\title{
Glass Delamination in Parenterals: A Brief Overview
}

Bindhu M Rayaprolu* and Jonathan J Strawser

PharmaForce Inc., Luitpold Pharmaceuticals Inc., OH, USA

*Corresponding author: Bindhu M Rayaprolu, PharmaForce Inc., A Subsidiary of Luitpold Pharmaceuticals Inc., 6610 New Albany Road East, OH 43054, USA, E-mail: bindhu.rayaprolu@gmail.com

Received date: Jun 30, 2017; Accepted date: Jul 03, 2017; Publish date: Jul 13, 2017

Copyright: (c) 2017 Rayaprolu BM, et al. This is an open-access article distributed under the terms of the Creative Commons Attribution License, which permits unrestricted use, distribution, and reproduction in any medium, provided the original author and source are credited.

\section{Editorial}

Packaging of parenteral formulations is a critical step in the product development process and most commonly achieved by using a glass container due to its relative inertness. Historically, the pharmaceutical market has utilized glass vials made from borosilicate and soda lime glass as preferred storage of pharmaceutical preparations. A potential issue with glass containers is glass delamination. Glass delamination results from surface degradation and subsequent glass chipping which is observed visually as glass flakes or lamella within the drug product solution. Though clinical relevance of visual particulate, including glass lamella, is difficult to ascertain, glass delamination should be taken seriously as any foreign particulate injected may be hazardous. The issue of glass delamination has caused several pharmaceutical manufacturers to voluntarily recall their drug products. As glass delamination might take multiple years to develop, pharmaceutical companies struggle to identify potential risky drug products in the early stages of development process and may be severely impacted due to occurrence while marketing. Financial burdens, both direct and indirect, may accrue if the drug product is actively marketed and subsequently recalled. Hence, the Food and Drug Administration recommends conducting stability studies of the pharmaceutical drug product with the intended packaging components. Glass manufacturers are also conducting new techniques and statistical control testing to determine the delamination propensity of the glass vials [1].

For parenteral formulations, Type I glass is the most commonly used packaging container. Two common types of Type I glass are available: Molded and Tubular glass. Molded glass containers are manufactured using single heat cycle and contain more alkali content than silicon, promoting greater chemical homogeneity [2]. Tubular glass containers are manufactured using two heat cycles where the tubing is made initially and then converted to mold. Careful consideration is to be given during this second step to obtain an interior surface which is resistant to chemical attack. Tubular glass containers have higher chemical resistance when compared to molded glass vials.

Important factors which may impact pharmaceutical glass durability include different chemical composition of the bulk glass, glass processing conditions, including temperature, forming, and annealing. Post glass production, other factors such as surface treatments, depyrogenation, terminal sterilization, and drug product material characteristics in contact with the container during its shelf life may impact durability. Differences in the chemical composition may also lend to significant changes in the melting temperature and the processing conditions required while forming the glass. For example, glass containing more silicon requires more heat to shape the container. Glass delamination usually occurs in the heel and shoulder region of the vial where extensive flaming occurs causing the evaporation of alkali and borates. This creates heterogeneous regions and potentially an exposed silica layer which may contribute to drug product interaction causing the release of glass flakes. As the glass is exposed to water, the silicon oxide will leach into the product thus leading to a less resistant glass. Characteristics of drug product solution in contact with the glass may also impact glass delamination. Acidic solutions cause the diffusion of water and exchange of hydrogen ions with the alkali ions such as sodium and potassium ions. Highly basic solutions $(\geq 8)$ cause the dissolution of silicon oxide layer. Ammonium sulfate treatment of vials is a process to remove the excess alkali from the surface which can also cause delamination [3,4]. Also, presence of buffers in the drug product solution, such as phosphates and citrates, can interact with the glass vials causing not only dissolution of elements present in the glass but also interact with the glass causing the delamination. Terminal sterilization of the drug product post filling may accelerate surface degradation and support increase likelihood for glass delamination. Glass delamination can also be caused during the storage of drug product at elevated temperatures. Products stored at room temperature have a higher propensity for delamination when compared to products stored at refrigerated conditions. Storage of glass in humid conditions and processing conditions such as depyrogenation in the presence of water vapour also shows reduced chemical resistance.

Careful consideration is to be given while selecting a glass vial for the drug product storage in an attempt to minimize glass delamination. The chosen drug product container closure should be able to protect the drug stability and also minimize the risk of delamination or glass chipping. There are several techniques to analyze glass delamination which includes optical microscopy, Scanning Electron Microscopy (SEM), Transmission Electron Microscopy (TEM), Secondary Ion Mass Spectrometry (SIMS) and Inductively Coupled Plasma Mass Spectrometry (ICP-MS). Light microscopy is used to examine the intact vials and determine if there is any presence of glass flakes in the drug product. SEM Analyzes the surface degradation of glass surfaces to detect flakes or pitting defects. TEM is advantageous to analyze thin delamination flakes. SIMS analyzes the hydrogen diffusion into glass. ICP-MS measures the trace levels of glass components such as Sodium, Boron, Aluminum, Calcium etc. in the drug product solutions. SEM coupled with energy dispersive X-ray spectrometry (SEM/EDS) is used to determine if the flakes are similar to that observed with glass delamination. SCHOTT Pharma has developed a delamination package which includes a detailed process to identify the delamination which is aligned with USP $<1660>$ guidanceEvaluation of the inner surface durability of glass containers. Surface glass test evaluates the internal surface of glass containers using water as an extraction medium. However, a lower value does not always indicate a durable inner surface. 
Citation: Rayaprolu BM, Strawser JJ (2017) Glass Delamination in Parenterals: A Brief Overview. J Pharmacovigil 5: e171. doi:

Page 2 of 2

Understanding the potential drug product interactions and impact processing a glass vial may have on a product shelf-life should be immediately evaluated at the onset of development. Experience within the pharmaceutical industry has generated ways to protect drug products while maintaining a stable environment. Quartz coating of the glass prevents the diffusion of ions from the glass and also provides a homogenous barrier between the glass surface and drug product. Molded glass contains lower silicon and a higher alkali and alkaline earth metal content and has a reduced risk of glass delamination. Careful control during processing of tubular vials also reduces the risk of glass delamination [5]. Polymeric primary container closure can be considered an option; however the ability to protect from oxygen, moisture, and leaching may become major concerns. Since this phenomenon might take several years to develop, careful selection of the container closure during product development and accelerated and long term stability studies are critical. Drug product manufacturers are encouraged to establish acceptable quality levels based on considerations for the aforementioned risks when selecting a glass manufacturing vendor for their parenteral drug products.

\section{References}

1. US Pharmacopeial Convention (2012) USP general chapter $<1660>$ evaluation of the inner surface durability of glass containers.

2. Haines D (2013) Evaluating parenteral packaging for glass delamination. Pharm Tech 37.

3. Guadagnino E, Zuccato D (2012) Delamination propensity of pharmaceutical glass containers by accelerated testing with different extraction media. PDA J Pharm Sci Technol 66: 116-125.

4. Haines D, Scheumann V, Rothhaar U (2013) Pre-testing stops a big problem before it even starts. Schott Pharma Services.

5. Food and Drug Administration (2016) Advisoryvgs. 